Original

\title{
Prevalencia de los factores de riesgo cardiovascular en trabajadores de una planta metalúrgica
}

\section{Prevalence of cardiovascular risk factors among workers in a metallurgical factory}

\section{Juan Antonio Alonso Díaz ', Ana Belén Calleja Méndez ', Soledad Borbolla Ruiz ${ }^{2}$}

1 Unidad Básica de Salud. Teka Industrial, S.A. Santander. España

2 Teka Industrial, S.A. Santander. España

Recibido: 24-07-12

Aceptado: 08-08-12

\section{Correspondencia}

Juan Antonio Alonso Díaz.

Teka Industrial, S. A.

Cajo, 17.

39011 Santander. España.

Teléfono: 942355050 - 510.

Fax: 942328849

Correo electrónico: jalonso@teka.com

Resumen

Objetivo: El estudio pretende conocer la prevalencia y la distribución de los factores de riesgo cardiovascular en una población concreta para poder implementar las medidas preventivas necesarias con criterios de eficiencia.

Métodos: Estudio descriptivo transversal de la distribución de los factores de riesgo cardiovascular mayores (dislipemia, tabaquismo, hipertensión arterial y diabetes mellitus) y subyacentes (sobrepeso/obesidad, sedentarismo, hipertrigliceridemia, consumo de alcohol y antecedentes familiares) en función de la edad y el sexo obtenidos mediante la aplicación de un protocolo estandarizado efectuado durante los reconocimientos médicos laborales periódicos de un grupo de 358 trabajadores, 330 varones y 28 mujeres, de una industria metalúrgica de Cantabria con una edad media de $42 \pm 8$ años.

Resultado: La dislipemia constituye el factor de riesgo más relevante por su elevada prevalencia (52\%) en una población relativamente joven, seguido del tabaquismo (35\%) y de la obesidad (22\%). Estos tres factores de riesgo son también los que presentan un riesgo atribuible mayor en la incidencia de cardiopatía isquémica en la población general española, por lo que deben ser objeto de intervención.

Conclusiones. Los servicios de salud laboral de las empresas tienen una posición privilegiada por su accesibilidad para evaluar y tratar los factores de riesgo cardiovascular presentes en la población trabajadora a la que sirven.

Med Segur Trab (Internet) 2012; 58 (228) 269-281

Palabras claves: Factores de riesgo cardiovascular. Salud laboral. 


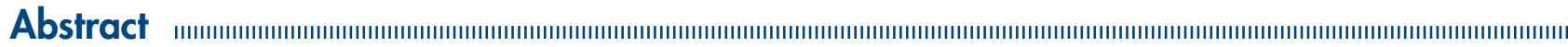

Objective: The study aims to determine the prevalence and distribution of cardiovascular risk factors in a specific population in order to implement the necessary preventative measures with efficiency criterias.

Methods: Cross-sectional study of the distribution of major cardiovascular risk factors (dyslipidemia, smoking, hypertension and diabetes mellitus) and the underlying factors (overweight / obesity, physical inactivity, hypertriglyceridemia, alcohol consumption and family history) as a function of age and sex obtained by applying a standardized protocol performed during periodic medical examinations at work to a group of 358 workers, 330 men and 28 women, in a metallurgical industry in Cantabria with a mean age of $42 \pm 8$ years.

Result: Dyslipidemia is the most important risk factor for its high prevalence (52\%) in a relatively young population, followed by smoking (35\%) and obesity (22\%). These three risk factors are also those with a higher attributable risk in the incidence of ischemic heart disease in the general Spanish population, so they should be subject to intervention.

Conclusions: Occupational Health Service companies have a privileged position due to their accessibility to evaluate and treat cardiovascular risk factors present in the working population they serve.

Med Segur Trab (Internet) 2012; 58 (228) 269-281

Key words: Cardiovascular risk factors. Occupational Health. 


\section{INTRODUCCIÓN}

Las enfermedades cardiovasculares constituyen la primera causa de morbimortalidad en los países industrializados entre los que se encuentra España. El descubrimiento del origen multifactorial de dichas enfermedades y la posibilidad de intervenir sobre los factores de riesgo modificables hace necesario su caracterización precisa para poder implementar protocolos de intervención de cuya efectividad existe una evidencia científica suficientemente avalada por la clínica y la epidemiología. El objetivo del presente estudio es conocer la distribución y la prevalencia de los factores de riesgo cardiovascular en una población concreta para diseñar e implementar estrategias preventivas eficientes tanto a nivel individual como colectivo.

\section{MATERIAL Y MÉTODO}

El estudio de los factores de riesgo cardiovascular se realizó con los datos obtenidos durante los reconocimientos médicos laborales de carácter periódico efectuados en 2011 a los trabajadores de una planta metalúrgica de Cantabria. La muestra objeto del estudio estuvo formada por 358 trabajadores, 330 varones y 28 mujeres. Todas las pruebas y los procedimientos utilizados fueron realizados tras la obtención del consentimiento informado para la realización de dichos exámenes de salud por parte de todos los trabajadores incluidos en el estudio.

El estudio es de corte transversal. En todos los individuos estudiados se aplicó un protocolo estandarizado que incluía la realización de una anamnesis estructurada, exploración física general y pruebas complementarias específicas, recogiéndose, además de las variables constitucionales (edad, sexo, peso, talla), otros datos relativos a los factores de riesgo cardiovascular presentes en cada individuo. Para su análisis posterior los factores de riesgo cardiovascular se dividieron, siguiendo las recomendaciones del "Word Heart and Stroke Forum" en factores de riesgo mayores, considerados así por ser causa directa de enfermedad cardiovascular, y factores subyacentes, que actúan sobre todo a través de los factores de riesgo mayores, aunque también de forma independiente, y, en general, no se incluyen en las ecuaciones de predicción del riesgo aunque sí se tienen en cuenta a la hora de ponderar ese riesgo en una persona determinada. Los factores de riesgo emergentes fueron excluidos del estudio. Entre los factores mayores clásicos de riesgo se incluye la hipertensión arterial (HTA), la dislipemia, la diabetes y el tabaquismo. Los factores subyacentes estudiados fueron la obesidad, el sedentarismo, el consumo de alcohol, la hipertrigliceridemia y la historia familiar de enfermedad cardiovascular prematura.

Las medidas antropométricas se obtuvieron con el trabajador de pie, descalzo y sin ropa en el tronco. La determinación de la tensión arterial se realizó según el procedimiento y la técnica estándar mediante un esfigmomanómetro aneroide Riester big ben ${ }^{\circledR}$ round. Para los análisis bioquímicos se realizaron extracciones de sangre venosa en ayunas, siendo las muestras remitidas al laboratorio acreditado de referencia, en donde fueron analizadas utilizando el método de oxidación para las determinaciones de la glucemia y el método enzimático para las determinaciones del colesterol total, HDL-colesterol y triglicéridos. El LDL-colesterol fue obtenido a partir de la fórmula de Friedewald, cuando los triglicéridos fueron inferiores a $400 \mathrm{mg} / \mathrm{dl}$. No se realizaron todas las mediciones tensionales necesarias para el diagnóstico de hipertensión arterial ni las determinaciones analíticas adicionales requeridas para el diagnóstico de diabetes mellitus y para iniciar tratamiento hipolipemiante en el caso de las hiperlipemias.

Se consideraron hipertensos los individuos con cifras de tensión arterial sistólica (TAS) $\geq 140 \mathrm{mmHg}$ o con tensión arterial diastólica (TAD) $\geq 90 \mathrm{mmHg}$ en el momento del reconocimiento y los que manifestaron padecer hipertensión arterial (HTA) o ya recibían tratamiento antihipertensivo. Se consideró HTA grado 1 a valores de 140-159 de TAS y/o 90-99 de TAD, HTA grado 2 a valores de 160-179 de TAS y/o 100-109 de TAD e HTA grado 
3 a valores de $\geq 180$ de TAS y/o $\geq 110$ de TAD. Se calificó de hipertensión sistólica aislada los valores $\geq 140$ de TAS y TAD $<90$.

Fueron considerados afectos de hipercolesterolemia (HC) los individuos con valores de colesterol total (CT) $\geq 200 \mathrm{mg} / \mathrm{dl}$ y aquellos que manifestaron esta enfermedad como antecedente o estaban en tratamiento dietético o farmacológico. Para el LDL-colesterol (c-LDL) se ha tomado como valor límite $<130 \mathrm{mg} / \mathrm{dl} \mathrm{y} \mathrm{no} \mathrm{se} \mathrm{aplicó} \mathrm{la} \mathrm{fórmula} \mathrm{de}$ Friedewald en 12 trabajadores porque los niveles de triglicéridos eran iguales o superiores a $400 \mathrm{mg} / \mathrm{dl}$. En el caso del HDL-colesterol sérico (c-HDL) las concentraciones $<40 \mathrm{mg}$ / dl en varones y $<45 \mathrm{mg} / \mathrm{dl}$ en mujeres $\mathrm{y} \geq 150 \mathrm{mg} / \mathrm{dl}$ para los triglicéridos (TG) sirven como marcadores de un riesgo cardiovascular aumentado.

Con relación a la diabetes mellitus (DM), se incluyeron como cifras de glucemia plasmática elevadas a efectos epidemiológicos los casos con glucemia basal $\geq 100 \mathrm{mg} / \mathrm{dl}$ y los que manifestaron padecer la enfermedad o estar en tratamiento con antidiabéticos orales (ADO) o insulina.

A efectos de clasificación se consideró fumador a los que manifestaron un consumo regular de tabaco en cualquiera de sus formas en el momento del reconocimiento médico o en los doce meses inmediatamente anteriores al examen de salud.

Para cuantificar la presencia de sobrepeso/obesidad se utilizó el índice de masa corporal (IMC o índice de Quetelec), resultado de dividir el peso en kg entre la talla en $\mathrm{m}^{2}$. Se consideró sobrepeso un IMC de 25 a 29 , obesidad si el IMC es $\geq 30$ y obesidad mórbida si es $\geq$ de 40 . Se consideró la presencia de obesidad abdominal cuando el perímetro abdominal es $\geq 102 \mathrm{~cm}$ en varones $\mathrm{y} \geq 88$ en mujeres.

El sedentarismo fue definido como la ausencia de un mínimo de tres episodios semanales de actividad física en tiempo de ocio de, al menos, 30 minutos de duración.

Para cuantificar el consumo de alcohol se ha utilizado el método ISCA (Interrogatorio Sistematizado de Consumos Alcohólicos). Sus resultados se expresan en Unidades de Bebida Estándar (UBE) semanales. La UBE es el contenido medio de alcohol de una bebida habitual y en la población española se ha establecido en 10 gr. Una ingesta superior a $5 \mathrm{UBE} /$ día se categoriza como factor de riesgo para el accidente cerebrovascular.

Se consideró como antecedente familiar de riesgo la historia de eventos cardiovasculares precoces en familiares de primer grado (padres o hermanos), en varones menores de 55 años y en mujeres menores de 65 años.

Conocidos los factores de riesgo cardiovascular presentes en cada uno de los trabajadores objeto del estudio, se procedió a cuantificar dicho riesgo mediante las tablas del proyecto SCORE (Systematic Coronary Risk Evaluation) calibradas para España. Las tablas de riesgo cardiovascular permiten, además de priorizar las intervenciones y motivar al paciente en el cumplimiento terapéutico, decidir cuando un paciente, en función de su grado de riesgo cardiovascular, debe recibir un tratamiento antihipertensivo o hipolipemiante en aquellos casos en los que la elevación de dichos factores individualmente considerados es moderada ${ }^{1}$. Las tablas del proyecto SCORE, cuya utilización está avalada por las Sociedades Europeas (Guía Europea de Prevención Cardiovascular en la Práctica Clínica) y el Comité Español Interdisciplinario para la Prevención Cardiovascular (CEIPC), estiman el riesgo de mortalidad cardiovascular a los 10 años e incluyen muertes coronarias y de origen cerebrovascular. Se consideró riesgo cardiovascular alto cuando el riesgo calculado según las tablas del SCORE fue igual o superior al 5\%, riesgo cardiovascular moderado cuando se obtuvieron valores del 3-4\% y riesgo cardiovascular bajo cuando los valores calculados según las tablas del SCORE fueron inferiores al 3\%. Los menores de 40 años fueron incluidos sistemáticamente en el primer grupo de edad de las tablas del SCORE. 


\section{RESULTADOS}

Se analizaron los datos de un total de 358 trabajadores, 330 varones (92\%) y 28 mujeres (8\%). La edad media de la población estudiada fue de $42 \pm 8$ años, con valores extremos de 25 y 62 años (tabla I), estando el 82\% de los individuos entre los 31 y los 50 años en el momento del estudio. En el grupo de varones, por representar la inmensa mayoría de los casos, la edad media fue la misma que en el total de la población, mientras que en el grupo de mujeres la edad media fue de $40 \pm 6$ años.

Tabla I. Distribución de la población objeto de estudio por edad y sexo

\begin{tabular}{l|r|r|r|r|r|r}
\hline \multirow{2}{*}{ Sexo } & \multicolumn{2}{|c|}{ Varones } & \multicolumn{2}{c|}{ Mujeres } & \multicolumn{2}{c}{ Total } \\
\cline { 2 - 7 } & $\mathbf{n}$ & $\%$ & $\mathbf{n}$ & \multicolumn{1}{c}{$\%$} & $\mathbf{n}$ & $\%$ \\
\hline Franja etaria & & & & & & \\
$\leq 30$ & 14 & 4 & 0 & 0 & 14 & 4 \\
$31-40$ & 138 & 42 & 16 & 57 & 154 & 43 \\
$41-50$ & 131 & 40 & 9 & 32 & 140 & 39 \\
$>50$ & 47 & 14 & 3 & 11 & 50 & 14 \\
\hline Total & 330 & 100 & 28 & 100 & 358 & 100 \\
\hline
\end{tabular}

La prevalencia y la distribución por sexos de los factores de riesgo mayores y subyacentes se recogen en las tablas II y III respectivamente. La tabla IV muestra la prevalencia y la distribución de los factores de riesgo según la edad.

Tabla II. Prevalencia y distribución por sexo de los factores de riesgo mayores

\begin{tabular}{|c|c|c|c|c|c|c|}
\hline \multirow{2}{*}{ Sexo } & \multicolumn{2}{|c|}{ Varones } & \multicolumn{2}{|c|}{ Mujeres } & \multicolumn{2}{|c|}{ Total } \\
\hline & n & $\%$ & $\mathbf{n}$ & $\%$ & n & $\%$ \\
\hline \multicolumn{7}{|l|}{ Tensión arterial } \\
\hline Normal & 284 & 86 & 25 & 89 & 309 & 86 \\
\hline HTA Grado 1 (TAS 140-159 y/o TAD 90-99) & 27 & 8 & 3 & 11 & 30 & 8 \\
\hline HTA Grado 2 (TAS 160-179 y/o TAD 100-109) & 8 & 2 & 0 & 0 & 8 & 2 \\
\hline HTA Grado 3 (TAS $\geq 180$ y/o TAD $\geq 110$ ) & 0 & 0 & 0 & 0 & 0 & 0 \\
\hline HTA Sistólica (TAS $\geq 140$ y TAD < 90) & 11 & 3 & 0 & 0 & 11 & 3 \\
\hline Total & 330 & 100 & 28 & 100 & 358 & 100 \\
\hline Pacientes hipertensos & 62 & 19 & 3 & 11 & 65 & 18 \\
\hline \multicolumn{7}{|l|}{ Colesterol total } \\
\hline Deseable $(<200)$ & 170 & 52 & 16 & 57 & 186 & 52 \\
\hline Limítrofe (200-239) & 110 & 33 & 8 & 29 & 118 & 33 \\
\hline Alto $(\geq 240)$ & 50 & 15 & 4 & 14 & 54 & 15 \\
\hline Total & 330 & 100 & 28 & 100 & 358 & 100 \\
\hline Pacientes hipercolesterolémicos & 172 & 52 & 13 & 46 & 185 & 52 \\
\hline \multicolumn{7}{|l|}{ LDL-Colesterol } \\
\hline Deseable $(<130)$ & 188 & 59 & 21 & 75 & 209 & 60 \\
\hline Limítrofe (130-159) & 86 & 27 & 4 & 14 & 90 & 26 \\
\hline Alto $(\geq 160)$ & 44 & 14 & 3 & 11 & 47 & 14 \\
\hline Total & 318 & 100 & 28 & 100 & 346 & 100 \\
\hline \multicolumn{7}{|l|}{ HDL-Colesterol } \\
\hline Bajo $(<40 \hat{\jmath} ;<45+)$ & 105 & 32 & 3 & 11 & 108 & 30 \\
\hline
\end{tabular}




\begin{tabular}{|c|c|c|c|c|c|c|}
\hline \multirow{2}{*}{ Sexo } & \multicolumn{2}{|c|}{ Varones } & \multicolumn{2}{|c|}{ Mujeres } & \multicolumn{2}{|c|}{ Total } \\
\hline & $\mathbf{n}$ & $\%$ & $\mathbf{n}$ & $\%$ & $\mathbf{n}$ & $\%$ \\
\hline Alto $(\geq 60)$ & 39 & 12 & 14 & 50 & 53 & 15 \\
\hline Total & 330 & 100 & 28 & 100 & 358 & 100 \\
\hline \multicolumn{7}{|l|}{ Glucemia } \\
\hline Normal $(<100)$ & 267 & 81 & 24 & 86 & 291 & 81 \\
\hline Glucosa basal alterada (100-125) & 52 & 16 & 4 & 14 & 56 & 16 \\
\hline Diabetes $(\geq 126)$ & 11 & 3 & 0 & 0 & 11 & 3 \\
\hline Total & 330 & 100 & 28 & 100 & 358 & 100 \\
\hline Pacientes diabéticos & 18 & 5 & 1 & 4 & 19 & 5 \\
\hline \multicolumn{7}{|l|}{ Tabaquismo } \\
\hline Actual & 120 & 36 & 4 & 14 & 124 & 35 \\
\hline Previo & 55 & 17 & 6 & 21 & 61 & 17 \\
\hline Nunca & 155 & 47 & 18 & 64 & 173 & 48 \\
\hline Total & 330 & 100 & 28 & 100 & 358 & 100 \\
\hline
\end{tabular}

Tabla III. Prevalencia y distribución por sexo de los factores de riesgo subyacentes

\begin{tabular}{|c|c|c|c|c|c|c|}
\hline \multirow{2}{*}{ Sexo } & \multicolumn{2}{|c|}{ Varones } & \multicolumn{2}{|c|}{ Mujeres } & \multicolumn{2}{|c|}{ Total } \\
\hline & $\mathbf{n}$ & $\%$ & $\mathbf{n}$ & $\%$ & $\mathbf{n}$ & $\%$ \\
\hline \multicolumn{7}{|l|}{ Índice de masa corporal } \\
\hline Bajo peso $($ IMC < 18,50) & 0 & 0 & 0 & 0 & 0 & 0 \\
\hline Normal (IMC 18,50-24) & 82 & 25 & 23 & 82 & 105 & 29 \\
\hline Sobrepeso (IMC 25-29) & 172 & 52 & 3 & 11 & 175 & 49 \\
\hline Obesidad Grado I (IMC 30-34) & 63 & 19 & 1 & 4 & 64 & 18 \\
\hline Obesidad Grado II (IMC 35-39) & 13 & 4 & 0 & 0 & 13 & 4 \\
\hline Obesidad Mórbida (IMC $\geq 40$ ) & 0 & 0 & 1 & 4 & 1 & 0 \\
\hline Total & 330 & 100 & 28 & 100 & 358 & 100 \\
\hline \multicolumn{7}{|l|}{ Perímetro abdominal } \\
\hline Normal $(\overbrace{}^{\lambda}<102 ;$ q $<88)$ & 249 & 75 & 24 & 86 & 273 & 76 \\
\hline Aumentado $(\hat{\partial} \geq 102 ;$; $\geq 88)$ & 81 & 25 & 4 & 14 & 85 & 24 \\
\hline Total & 330 & 100 & 28 & 100 & 358 & 100 \\
\hline \multicolumn{7}{|l|}{ Sedentarismo } \\
\hline Presente & 127 & 38 & 19 & 68 & 146 & 41 \\
\hline Ausente & 203 & 62 & 9 & 32 & 212 & 59 \\
\hline Total & 330 & 100 & 28 & 100 & 358 & 100 \\
\hline \multicolumn{7}{|l|}{ Triglicéridos } \\
\hline Normal $(<150)$ & 204 & 62 & 25 & 89 & 229 & 64 \\
\hline Limítrofe (150-199) & 50 & 15 & 1 & 4 & 51 & 14 \\
\hline Elevado (200-499) & 70 & 21 & 2 & 7 & 72 & 20 \\
\hline Muy elevado $(\geq 500)$ & 6 & 2 & 0 & 0 & 6 & 2 \\
\hline Total & 330 & 100 & 28 & 100 & 358 & 100 \\
\hline \multicolumn{7}{|l|}{ Consumo de alcohol } \\
\hline De riesgo cardiovascular (> 5 UBE/día) & 10 & 3 & 0 & 0 & 10 & 3 \\
\hline No riesgo cardiovascular ( $\leq 5 \mathrm{UBE} /$ día) o no consumo & 320 & 97 & 28 & 100 & 348 & 97 \\
\hline Total & 330 & 100 & 28 & 100 & 358 & 100 \\
\hline
\end{tabular}




\begin{tabular}{|c|c|c|c|c|c|c|}
\hline \multirow{2}{*}{ Sexo } & \multicolumn{2}{|c|}{ Varones } & \multicolumn{2}{|c|}{ Mujeres } & \multicolumn{2}{|c|}{ Total } \\
\hline & n & $\%$ & $\mathbf{n}$ & $\%$ & n & $\%$ \\
\hline Positivos & 27 & 8 & 1 & 4 & 28 & 8 \\
\hline Negativos & 303 & 92 & 27 & 96 & 330 & 92 \\
\hline Total & 330 & 100 & 28 & 100 & 358 & 100 \\
\hline
\end{tabular}

Tabla IV. Prevalencia y distribución de los factores de riesgo con relación a la edad

\begin{tabular}{|c|c|c|c|c|c|c|c|c|c|c|}
\hline \multirow{2}{*}{ Edad } & \multicolumn{2}{|c|}{$\leq 30$} & \multicolumn{2}{|c|}{$31-40$} & \multicolumn{2}{|c|}{$41-50$} & \multicolumn{2}{|c|}{$>50$} & \multicolumn{2}{|c|}{ Total } \\
\hline & $\mathbf{n}$ & $\%$ & n & $\%$ & n & $\%$ & n & $\%$ & $\mathbf{n}$ & $\%$ \\
\hline \multicolumn{11}{|l|}{ Sexo } \\
\hline Varones & 14 & 100 & 138 & 90 & 131 & 94 & 47 & 94 & 330 & 92 \\
\hline Mujeres & 0 & 0 & 16 & 10 & 9 & 6 & 3 & 6 & 28 & 8 \\
\hline Total & 14 & 100 & 154 & 100 & 140 & 100 & 50 & 100 & 358 & 100 \\
\hline
\end{tabular}

Tensión arterial

\begin{tabular}{|c|c|c|c|c|c|c|c|c|c|c|}
\hline Normal & 11 & 79 & 142 & 92 & 118 & 84 & 38 & 76 & 309 & 86 \\
\hline HTA & 3 & 21 & 12 & 8 & 22 & 16 & 12 & 24 & 49 & 14 \\
\hline Total & 14 & 100 & 154 & 100 & 140 & 100 & 50 & 100 & 358 & 100 \\
\hline Pacientes hipertensos & 3 & 21 & 12 & 8 & 28 & 20 & 22 & 44 & 65 & 18 \\
\hline
\end{tabular}

\section{Colesterol}

\begin{tabular}{lrrrrrrrrrr} 
Deseable & 10 & 71 & 87 & 56 & 66 & 47 & 23 & 46 & 186 & 52 \\
Elevado & 4 & 29 & 67 & 44 & 74 & 53 & 27 & 54 & 172 & 48 \\
\hline Total & 14 & 100 & 154 & 100 & 140 & 100 & 50 & 100 & 358 & 100 \\
\hline Pacientes hipercolesterolémicos & 4 & 29 & 68 & 44 & 77 & 55 & 36 & 72 & 185 & 52 \\
\hline
\end{tabular}

\section{Tabaquismo}

\begin{tabular}{lrrrrrrrrrr} 
Fumador & 7 & 50 & 49 & 32 & 47 & 34 & 21 & 42 & 124 & 35 \\
Ex ó no fumador & 7 & 50 & 105 & 68 & 93 & 66 & 29 & 58 & 234 & 65 \\
\hline Total & 14 & 100 & 154 & 100 & 140 & 100 & 50 & 100 & 358 & 100 \\
\hline
\end{tabular}

\section{Glucemia}

\begin{tabular}{lrrrrrrrrrr} 
Normal & 12 & 86 & 135 & 88 & 113 & 81 & 31 & 62 & 291 & 81 \\
Elevada & 2 & 14 & 19 & 12 & 27 & 19 & 19 & 38 & 67 & 19 \\
\hline Total & 14 & 100 & 154 & 100 & 140 & 100 & 50 & 100 & 358 & 100 \\
\hline Pacientes diabéticos & 0 & 0 & 3 & 2 & 6 & 4 & 10 & 20 & 19 & 5
\end{tabular}

\section{Índice de masa corporal}

\begin{tabular}{lrrrrrrrrrr} 
Normal & 7 & 50 & 54 & 35 & 37 & 26 & 7 & 14 & 105 & 29 \\
Sobrepeso y/o obesidad & 7 & 50 & 100 & 65 & 103 & 74 & 43 & 86 & 253 & 71 \\
\hline Total & 14 & 100 & 154 & 100 & 140 & 100 & 50 & 100 & 358 & 100
\end{tabular}

Con relación a la presión arterial, se han considerado hipertensos a un total de 65 trabajadores (18\%), de los que 49 (14\%) tenían valores de TA superiores a los considerados normales y 16 (4\%) estaban diagnosticados de hipertensión y con tratamiento farmacológico pero presentaban valores normales. Otros 11 trabajadores eran hipertensos en tratamiento y con valores elevados de TA, lo que hace un total de 27 trabajadores en tratamiento con fármacos antihipertensivos. En base a estos datos cabe destacar que solamente el $42 \%$ de los hipertensos (27 de 65) estaba en tratamiento farmacológico y, de ellos, solamente el 
59\% (16 de 27) están bien controlados. El 86\% de los trabajadores presentaron valores normales de tensión arterial y el $\mathbf{9 4 \%}$ de los que presentaron valores elevados fueron varones. Se evidenció un notable incremento del porcentaje de hipertensos en los grupos de mayor edad. Así, en el grupo de 31 a 40 años el porcentaje de hipertensos fue del 8\%, en el de 41 a 50 años del 20\% y en el de los mayores de 50 años del $44 \%$.

Se consideraron afectos de hipercolesterolemia los que presentaron valores de colesterol total $\geq 200 \mathrm{mg} / \mathrm{dl}$ en la analítica (172 individuos; 48\%) y los que habiendo obtenido valores normales estaban diagnosticados de la enfermedad (13 individuos; 4\%), lo que hace un total de 185 individuos con hipercolesterolemia (52\%). Otros 37 trabajadores ya diagnosticados presentaban cifras elevadas. De los 50 trabajadores diagnosticados de hipercolesterolemia, 26 (52\%) estaban con tratamiento farmacológico, de los cuales solamente 13 (50\%) estaban bien controlados.

Los valores de LDL-colesterol se obtuvieron mediante la fórmula de Friedewald sobre un total de 346 muestras de sangre debido a que en 12 casos no fue de aplicación porque los niveles de triglicéridos obtenidos eran iguales o superiores a $400 \mathrm{mg} / \mathrm{dl}$. El $60 \%$ de los trabajadores tenían valores considerados deseables $(<130 \mathrm{mg} / \mathrm{dl})$ y el $40 \%$ elevados ( $\geq 130 \mathrm{mg} / \mathrm{dl})$.

Se obtuvieron glucemias basales en el rango de la normalidad en 291 trabajadores (81\%), mientras que 56 (16\%) presentaban cifras compatibles con glucosa basal alterada y $11(3 \%)$ valores de rango diabético, sin diferencias significativas por sexo. En la población estudiada había ya 12 trabajadores diagnosticados de diabetes mellitus y en tratamiento con antidiabéticos orales (ADO) o insulina. De ellos, 2 presentaban glucemias inferiores a 100, 6 entre 100 y 125 y 4 superiores a 125. Se han considerado diabéticos a un total de 19 trabajadores (5\%), los 11 con glucemia basal igual o superior a $126 \mathrm{mg} / \mathrm{dl}$ y los 8 con valores de glucemia inferiores en el momento del análisis.

Respecto al hábito tabáquico cabe destacar que un 35\% de los trabajadores encuestados eran fumadores activos o con menos de un año de abandono del hábito, el 97\% de los cuales eran varones, sin apreciarse diferencias significativas en función de los grupos de edad.

Con relación a la obesidad, se obtuvieron datos relativos al IMC y al perímetro abdominal. Respecto al IMC cabe señalar que el 29\% de los trabajadores presentó resultados normales, el $49 \%$ sobrepeso y el $22 \%$ restante obesidad. La prevalencia del sobrepeso y de la obesidad fue claramente superior en el grupo de varones, y su incremento porcentual fue paralelo a la edad. Según el perímetro abdominal, se apreció obesidad central en el $24 \%$ de los casos. El porcentaje de obesos según el IMC, tanto para cada sexo como para el total de los trabajadores estudiados, mostró una estrecha correlación con los hallados aplicando el criterio de perímetro abdominal aumentado.

El sedentarismo estaba presente en el $41 \%$ de los trabajadores, especialmente en el grupo de mujeres y solamente el 3\% de los trabajadores encuestados admitía un consumo de alcohol en cantidades consideradas como de riesgo cardiovascular. Por último, se constataron antecedentes familiares de enfermedad cardiovascular precoz en el $8 \%$ de los trabajadores encuestados.

Con relación a la presencia de los distintos factores de riesgo analizados (tabla V), solamente $12 \%$ de los trabajadores incluidos en el estudio no presenta ninguno, siendo la proporción igual en varones y en mujeres. La distribución porcentual del número de factores de riesgo en el grupo de varones y en el de mujeres se muestra en el gráfico 1. El 54\% de los varones y el 78\% de las mujeres presentaban uno o dos factores de riesgo, mientras que tres o cuatro factores de riesgo estaban presentes en el $31 \%$ de los varones y en el $11 \%$ del grupo de mujeres. Por tanto, el grupo de varones presentó una distribución más uniforme del número de factores de riesgo, mientras que el grupo de mujeres mostró un máximo en uno-dos factores de riesgo y una disminución porcentual muy marcada a partir de tres o más factores de riesgo. 
Tabla V. Factores de riesgo presentes en la población estudiada

\begin{tabular}{|c|c|c|c|c|c|c|}
\hline \multirow{2}{*}{ Sexo } & \multicolumn{2}{|c|}{ Varones } & \multicolumn{2}{|c|}{ Mujeres } & \multicolumn{2}{|c|}{ Total } \\
\hline & n & $\%$ & $\mathbf{n}$ & $\%$ & $\mathbf{n}$ & $\%$ \\
\hline \multicolumn{7}{|l|}{ Número de factores de riesgo } \\
\hline Ninguno & 39 & 12 & 3 & 11 & 42 & 12 \\
\hline \multicolumn{7}{|l|}{ Uno } \\
\hline Dislipemia (D) & 56 & 17 & 2 & 7 & 58 & 16 \\
\hline Sedentarismo (S) & 10 & 3 & 9 & 32 & 19 & 5 \\
\hline Tabaco $(\mathrm{T})$ & 13 & 4 & 0 & 0 & 13 & 4 \\
\hline Diabetes Mellitus (DM) & 3 & 1 & 0 & 0 & 3 & 1 \\
\hline Obesidad (O) & 2 & 1 & 0 & 0 & 2 & 1 \\
\hline $\begin{array}{l}\text { Antecedentes familiares de enf. cardiovascular precoz } \\
\text { (AFECV) }\end{array}$ & 2 & 1 & 0 & 0 & 2 & 1 \\
\hline Hipertensión arterial (HTA) & 2 & 1 & 0 & 0 & 2 & 1 \\
\hline Total & 88 & 27 & 11 & 39 & 99 & 28 \\
\hline \multicolumn{7}{|l|}{ Dos } \\
\hline $\mathrm{D}+\mathrm{S}$ & 23 & 7 & 6 & 21 & 29 & 8 \\
\hline $\mathrm{D}+\mathrm{T}$ & 21 & 6 & 1 & 4 & 22 & 6 \\
\hline D+HTA & 14 & 4 & 0 & 0 & 14 & 4 \\
\hline Otros & 30 & 9 & 4 & 14 & 34 & 9 \\
\hline Total & 88 & 27 & 11 & 39 & 99 & 28 \\
\hline \multicolumn{7}{|l|}{ Tres } \\
\hline $\mathrm{D}+\mathrm{S}+\mathrm{T}$ & 33 & 10 & 0 & 0 & 33 & 9 \\
\hline $\mathrm{D}+\mathrm{T}+\mathrm{O}$ & 7 & 2 & 1 & 4 & 8 & 2 \\
\hline $\mathrm{D}+\mathrm{O}+\mathrm{S}$ & 7 & 2 & 0 & 0 & 7 & 2 \\
\hline Otros & 25 & 8 & 1 & 4 & 26 & 7 \\
\hline Total & 72 & 22 & 2 & 7 & 74 & 21 \\
\hline \multicolumn{7}{|l|}{ Cuatro } \\
\hline $\mathrm{D}+\mathrm{T}+\mathrm{S}+\mathrm{O}$ & 7 & 2 & 0 & 0 & 7 & 2 \\
\hline $\mathrm{D}+\mathrm{T}+\mathrm{S}+\mathrm{HTA}$ & 6 & 2 & 1 & 4 & 7 & 2 \\
\hline $\mathrm{D}+\mathrm{T}+\mathrm{S}+\mathrm{AFECV}$ & 3 & 1 & 0 & 0 & 3 & 1 \\
\hline Otros & 14 & 4 & 0 & 0 & 14 & 4 \\
\hline Total & 30 & 9 & 1 & 4 & 31 & 9 \\
\hline \multicolumn{7}{|l|}{ Cinco } \\
\hline $\mathrm{D}+\mathrm{HTA}+\mathrm{S}+\mathrm{O}+\mathrm{AFECV}$ & 3 & 1 & 0 & 0 & 3 & 1 \\
\hline $\mathrm{D}+\mathrm{HTA}+\mathrm{DM}+\mathrm{S}+\mathrm{O}$ & 2 & 1 & 0 & 0 & 2 & 1 \\
\hline $\mathrm{D}+\mathrm{HTA}+\mathrm{DM}+\mathrm{S}+\mathrm{T}$ & 1 & 0 & 0 & 0 & 1 & 0 \\
\hline Otros & 6 & 2 & 0 & 0 & 6 & 2 \\
\hline Total & 12 & 4 & 0 & 0 & 12 & 3 \\
\hline \multicolumn{7}{|l|}{ Seis } \\
\hline $\mathrm{D}+\mathrm{HTA}+\mathrm{DM}+\mathrm{S}+\mathrm{O}+\mathrm{AFECV}$ & 1 & 0 & 0 & 0 & 1 & 0 \\
\hline $\mathrm{D}+\mathrm{HTA}+\mathrm{DM}+\mathrm{S}+\mathrm{T}+\mathrm{AFECV}$ & 0 & 0 & 0 & 0 & 0 & 0 \\
\hline $\mathrm{D}+\mathrm{HTA}+\mathrm{DM}+\mathrm{S}+\mathrm{T}+\mathrm{O}$ & 0 & 0 & 0 & 0 & 0 & 0 \\
\hline Total & 1 & 0 & 0 & 0 & 1 & 0 \\
\hline
\end{tabular}


El factor de riesgo que más frecuentemente se presentó de forma aislada fue la dislipemia (16\%) y el sedentarismo (5\% del total, $32 \%$ en el grupo de mujeres). Los dos factores de riesgo más frecuentemente asociados fueron dislipemia y sedentarismo (8\%), especialmente en el grupo de mujeres (21\%), seguido de dislipemia y tabaquismo (6\%). Considerando tres factores de riesgo, la asociación más frecuente (9\%) fue la de dislipemia, tabaquismo y sedentarismo. Las distintas asociaciones de 4 o más factores de riesgo tienen una prevalencia máxima del 1\% en la población estudiada.

Gráfico 1 Distribución del número de factores de riesgo cardiovascular por sexos

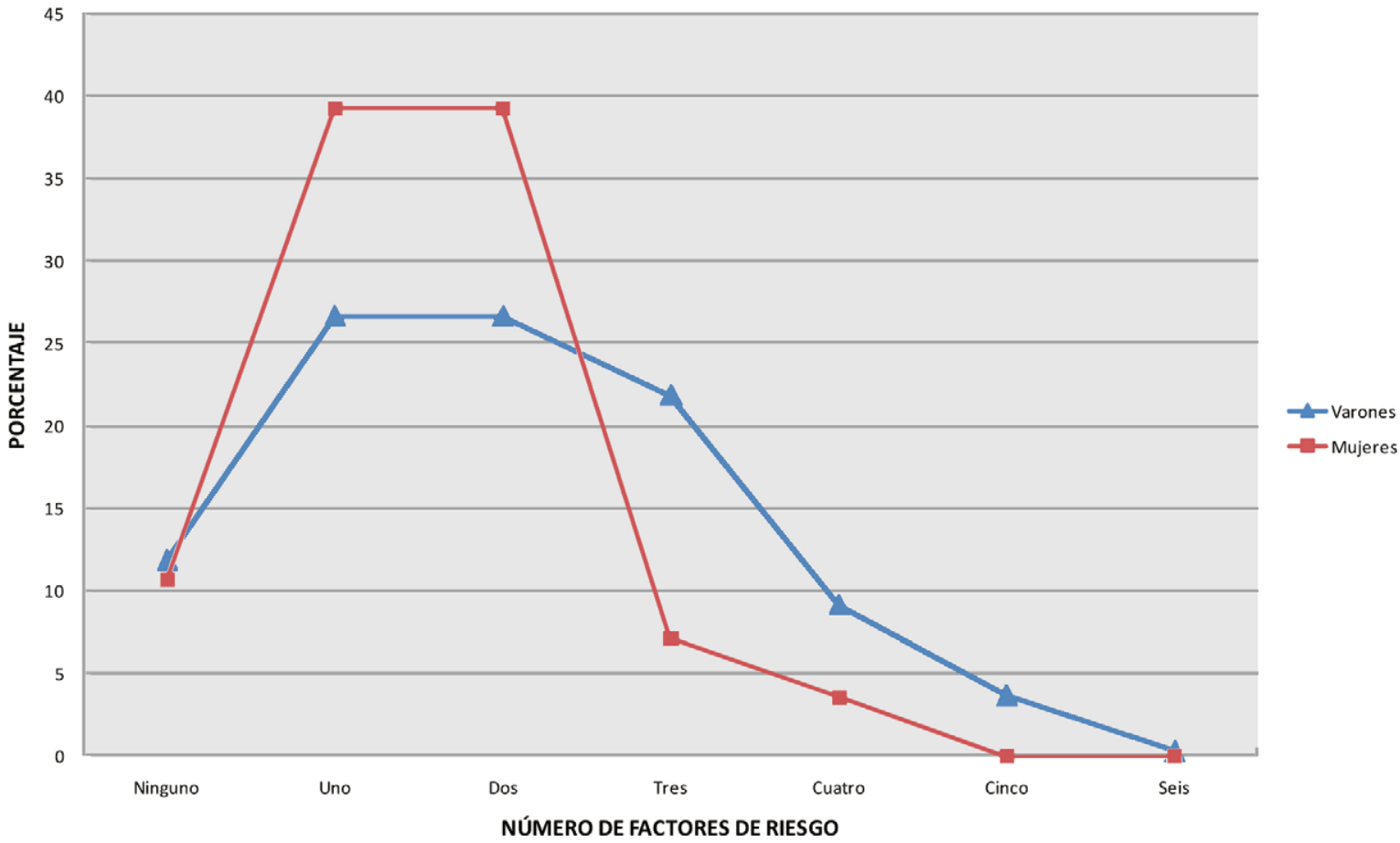

Con respecto a la distribución del grado de riesgo cardiovascular según el sexo y la edad (tabla VI), cabe señalar que 34 trabajadores (33 varones y 1 mujer) fueron excluidos de la evaluación mediante las tablas del SCORE y considerados de riesgo alto por presentar una o más de las siguientes características clínicas: diabetes mellitus, glucemia basal > $126 \mathrm{mg} / \mathrm{dl}$, enfermedad cardiovascular previa, colesterol total $>320 \mathrm{mg} / \mathrm{dl}$, LDL-colesterol $>240 \mathrm{mg} / \mathrm{dl}$ y/o consumo de más de 20 cigarrillos/día. Por tanto, fueron considerados con riesgo alto por su situación clínica el $9 \%$ de los trabajadores incluidos en el estudio. Del resto, a los que se valoró su grado de riesgo en función de las tablas del SCORE, un $1 \%$ presentó riesgo alto ( $>5 \%$ ), un $4 \%$ presentó riesgo moderado (3 - 4\%) y un 94\% presentó un riesgo bajo, sin que se evidencien diferencias significativas con respecto al sexo.

Por grupos de edad se muestra un aumento del grado de riesgo paralelo a la edad, tanto en los considerados con riesgo alto por su situación clínica ( $0 \%$ en menores de 31 años, $6 \%$ entre 31 y $40,9 \%$ entre 41 y 50 y $24 \%$ en mayores de 50 años), como en los evaluados por el SCORE (el 100\% de los trabajadores menores de 41 años presentaba riesgo bajo, el 95\% de los de 41 a 50 años y el 71\% de los mayores de 50 años). 
Tabla VI. Distribución del riesgo cardiovascular por sexo y edad

\begin{tabular}{|c|c|c|c|c|c|c|}
\hline \multirow{2}{*}{ Sexo } & \multicolumn{2}{|c|}{ Varones } & \multicolumn{2}{|c|}{ Mujeres } & \multicolumn{2}{|c|}{ Total } \\
\hline & $\mathrm{n}$ & $\%$ & $\mathrm{n}$ & $\%$ & $\mathrm{n}$ & $\%$ \\
\hline \multicolumn{7}{|l|}{ Riesgo cardiovascular } \\
\hline Alto (SCORE $\geq 5 \%$ ) & 4 & 1 & 0 & 0 & 4 & 1 \\
\hline Moderado (SCORE 3 - 4\%) & 14 & 5 & 0 & 0 & 14 & 4 \\
\hline Bajo (SCORE < 3\%) & 279 & 94 & 27 & 100 & 306 & 94 \\
\hline Total SCORE & 297 & 100 & 27 & 100 & 324 & 100 \\
\hline Alto por la clínica & 33 & 10 & 1 & 4 & 34 & 9 \\
\hline Total & 330 & 100 & 28 & 100 & 358 & 100 \\
\hline
\end{tabular}

\begin{tabular}{|c|c|c|c|c|c|c|c|c|c|c|}
\hline \multirow{2}{*}{ Edad } & \multicolumn{2}{|c|}{$\leq 30$} & \multicolumn{2}{|c|}{$31-40$} & \multicolumn{2}{|c|}{$41-50$} & \multicolumn{2}{|c|}{$>50$} & \multicolumn{2}{|c|}{ Total } \\
\hline & $\mathbf{n}$ & $\%$ & $\mathbf{n}$ & $\%$ & $\mathbf{n}$ & $\%$ & n & $\%$ & $\mathbf{n}$ & $\%$ \\
\hline \multicolumn{11}{|l|}{ Riesgo cardiovascular } \\
\hline Alto $($ SCORE $\geq 5 \%)$ & 0 & 0 & 0 & 0 & 0 & 0 & 4 & 11 & 4 & 1 \\
\hline Moderado (SCORE 3 - 4\%) & 0 & 0 & 0 & 0 & 7 & 5 & 7 & 18 & 14 & 4 \\
\hline Bajo (SCORE < 3\%) & 14 & 100 & 144 & 100 & 121 & 95 & 27 & 71 & 306 & 94 \\
\hline Total SCORE & 14 & 100 & 144 & 100 & 128 & 100 & 38 & 100 & 324 & 100 \\
\hline Alto por la clínica & 0 & 0 & 9 & 6 & 13 & 9 & 12 & 24 & 34 & 9 \\
\hline Total & 14 & 100 & 153 & 100 & 141 & 100 & 50 & 100 & 358 & 100 \\
\hline
\end{tabular}

\section{DISCUSIÓN}

El estudio puso de manifiesto la desigual distribución de los factores de riesgo cardiovascular y la elevada prevalencia de alguno de ellos en un grupo poblacional de edad media relativamente joven y mayoritariamente masculino. La escasa proporción de mujeres en la población objeto del estudio limita la posibilidad de extraer conclusiones válidas y fiables por sexos.

El orden de prevalencia en la población estudiada de los factores de riesgo cardiovascular es: hipercolesterolemia (52\%), sedentarismo (41\%), hipertrigliceridemia (36\%), tabaquismo (35\%), obesidad (22\%), hipertensión arterial (18\%), antecedentes familiares de riesgo (8\%), diabetes mellitus (5\%) y consumo de alcohol de riesgo (3\%).

La prevalencia de la hipercolesterolemia fue muy similar a la obtenida en grupos poblacionales españoles de edad media muy superior $(57 \pm 17 \text { años })^{3}$. Diferentes estudios han puesto de manifiesto que del $50 \%$ de dislipémicos presentes en la población general conoce que lo es y solamente 1 de cada 3 diagnosticados y tratados está controlado adecuadamente ${ }^{5-7}$. En nuestro estudio el $27 \%$ conocía su enfermedad y 1 de cada 4 estaba correctamente controlado. Se constató un aumento del número de pacientes dislipémicos con la edad, aunque su distribución fue más homogénea que en el caso de la hipertensión arterial. Cifras de HDL-colesterol menores de $40 \mathrm{mg} / \mathrm{dl}$ para los varones y menores de 45 $\mathrm{mg} / \mathrm{dl}$ para las mujeres, indicadoras de un riesgo cardiovascular aumentado ${ }^{8}$, se objetivaron en el $32 \%$ de los varones y en el $11 \%$ de las mujeres. Cifras de triglicéridos mayores de $150 \mathrm{mg} / \mathrm{dl}$ igualmente indicativas de riesgo cardiovascular aumentado ${ }^{8} \mathrm{se}^{\mathrm{s}}$ objetivaron en el 36\% de los casos, especialmente en varones. En nuestro estudio, la elevada prevalencia de dislipemia adquiere una relevancia mayor aún si se tiene en cuenta la baja edad media de la población estudiada, su elevada incidencia en la aparición de cardiopatía isquémica, siendo su tercera causa más frecuente ${ }^{9}$, y que es un factor de riesgo modificable y con efecto reversible. 
El sedentarismo estaba presente en el $41 \%$ de los encuestados, especialmente en el grupo de mujeres, igual a la proporción de inactivos en tiempo libre en España según la última Encuesta Nacional de Salud ${ }^{10}(40,3 \%)$.

La prevalencia del tabaquismo fue del 35\%, ligeramente inferior a la obtenida en otros estudios de población trabajadora ${ }^{14}$, pero el porcentaje de fumadores aumentó con la edad, invirtiéndose la tendencia observada en otros estudios de poblaciones con mayor edad media en los que se demuestra una disminución significativa del consumo de tabaco con la edad, especialmente en las mujeres ${ }^{3}$. Además de su elevada prevalencia en nuestra serie, el tabaquismo es el responsable del 34\% de los eventos de cardiopatía isquémica, y ocupa el segundo lugar como factor de riesgo poblacional ${ }^{9}$, lo que, unido a sus efectos perniciosos a múltiples niveles, hace que deba ser objeto de una intervención decidida tanto a nivel individual como de grupo.

El sobrepeso y la obesidad definidos según el IMC estaban presenten en el 49\% y en el $22 \%$ respectivamente de los individuos, valores prácticamente iguales a los obtenidos en otros estudios sobre factores de riesgo cardiovascular ${ }^{3-9}$. El exceso de peso es el principal causante de cardiopatía de origen coronario, responsable de más del $40 \%$ de los casos $^{9}$. El perímetro abdominal resultó aumentado en el $24 \%$ de los individuos, lo que evidencia una buena correlación entre la obesidad central y el IMC ${ }^{3}$ 30. La distribución por grupos de edad fue bastante homogénea, aunque con una tendencia a incrementar su prevalencia con la edad. Si bien la asociación de sobrepeso y riesgo coronario no está suficientemente aclarada, especialmente en varones ${ }^{9}$, precede en muchas ocasiones a la obesidad, que es un factor de riesgo que se asocia con frecuencia a dislipemia, hipertensión arterial, diabetes y aumento de la mortalidad por enfermedad cardiovascular, además de estar íntimamente relacionada con el sedentarismo, con el que comparte un mismo abordaje terapéutico.

La prevalencia de la hipertensión arterial en nuestro estudio fue del $18 \%$, mientras que en la población general española mayor de 18 años está alrededor del 36\% $\%^{2,3}$. Esta diferencia debe ser atribuida a la edad media de la población estudiada, dado que se constata una notable tendencia al incremento del porcentaje de hipertensos con la edad que aumenta en más del doble en cada década a partir de los 31 años. Existen también diferencias importantes con respecto a la población española en cuanto al conocimiento de la enfermedad y al tratamiento. Así, el 65\% de los hipertensos españoles sabe que lo es, el $85 \%$ está en tratamiento farmacológico y el $25 \%$ de los hipertensos tratados logra controlar su tensión arterial ${ }^{4}$, mientras que en nuestra serie solamente el $42 \%$ de los hipertensos sabía que lo era, todos los que se sabían hipertensos estaban en tratamiento farmacológico y el 59\% de ellos estaba bien controlado con la medicación.

La prevalencia de diabetes mellitus en nuestro estudio fue del 5\%, la mitad que en la población general española ${ }^{10}$, aunque su notable incremento con la edad hace que su prevalencia en nuestra serie llegue al 20\% en mayores de 50 años. El 63\% de los diabéticos presentes en la población estudiada conocían su enfermedad y estaban en tratamiento y de ellos el $67 \%$ presentaban glucemias inferiores a $126 \mathrm{mg} / \mathrm{dl}$. En la población general el grado de conocimiento de la diabetes es del $50 \%{ }^{10}$ y su control en atención primaria es inferior al 30\% cuando se considera la glucemia basal inferior a $126 \mathrm{mg} / \mathrm{dl}^{10-13}$.

Con relación a la elevada prevalencia de algunos de los factores de riesgo analizados, llama la atención que el riesgo cardiovascular global de la población estudiada según las tablas del proyecto SCORE sea bajo en el $94 \%$ de los casos, lo cual es debido fundamentalmente a que el riesgo absoluto está íntimamente vinculado a la edad, pero también a que no tiene en cuenta todos los factores de riesgo analizados y a la escasa prevalencia de la hipertensión arterial en la población estudiada. 


\section{CONCLUSIONES}

La necesaria priorización de las intervenciones terapéuticas comunitarias dirigidas a modificar los estilos de vida poco saludables deben estar dirigidas a grupos poblacionales específicos e incluir acciones relevantes sobre los principales factores de riesgo presentes en ellos, cuyo análisis previo como parte integrante de la vigilancia de la salud colectiva, permitirá implementar actividades de promoción de salud eficientes y evaluar sus resultados con criterios objetivos.

Los servicios médicos de salud laboral de las empresas, por su cercanía y accesibilidad, constituyen un recurso excelente y generalmente infrautilizado para la detección, seguimiento y control de los factores de riesgo cardiovascular por lo que deberían coordinarse con los recursos asistenciales al objeto de intervenir más eficientemente en los problemas de salud de la población trabajadora.

\section{REFERENCIAS BIBLIOGRÁFICAS}

1. González González AI, Obaya Rebollar JC. ¿Qué tabla es más adecuada para el cálculo del riesgo coronario en atención primaria? Jano 2009. No 1.744:19-24

2. Banegas J. Epidemiología de la hipertensión arterial en España. Situación actual y perspectivas. Hipertensión. 2005;22:253-362.

3. Gabriel R, Alonso M, Segura A, Tormo MJ, Artigao LM, Banegas JR y cols. Prevalencia, distribución y variabilidad geográfica en los principales factores de riesgo cardiovascular en España. Análisis agrupado de datos individuales de estudios epidemiológicos poblacionales: estudio ERICE. Rev Esp Cardiol 2008;61(10): 1030-40.

4. Banegas JR, Segura J, Ruilope LM, Luque M, García-Robles R, Campo C y cols. Blood presure control and physician management of hypertension in hospital hypertension units in Spain. Hypertension. 2004; $43: 1338-44$.

5. Vegazo O, Banegas J, Civeira F, Serrano P, Luengo E, Mantilla T. Prevalencia de las dislipemias en consultas ambulatorias del sistema sanitario español. Estudio Hispalipid. Med Clin (Barc). 2006;127:331-4.

6. Banegas JR, Vegazo O, Serrano P, Luengo E, Mantilla T, Fernández R y cols. The gap between dyslipidemia control perceived by physicians and objetive control patterns in Spain. Atherosclerosis. 2006; 188;420-4.

7. Rodríguez-Roca G, Llisterri J, Barrios V, Alonso-Moreno J, Banegas J, Lou S y cols. Grado de control de la dislipemia en los pacientes españoles asistidos en atención primaria. Estudio LIPICAD. Clin Invest Arterioscl. 2006;18:226-238.

8. Guía Europea de Prevención Cardiovascular en la Práctica Clínica. Adaptación española del CEIPP. Ministerio de Sanidad y Consumo. 2008.

9. Medrano M, Pastor-Barriuso R, Boix R, del Barrio JL, Damián J, Álvarez R, Martín A. Riesgo coronario atribuible a los factores de riesgo cardiovascular en población española. Rev Esp Cardiol. 2007;60(12):1250-6.

10. Ministerio de Sanidad y Consumo. Encuesta Nacional de Salud de España. Madrid. Ministerio de Sanidad y Consumo 2005. Disponible en http://www.msc.es/estadEstudios/estadisticas/home.htm

11. Orozco-Beltrán D, Gil-Guillén VF, Quirce F, Navarro-Pérez F, Pineda M. Control of diabetes and cardiovascular risck factors in patients with rype 2 diabetes in primary care. The gap between guidelines and reality in Spain. Int J Clin Prac. 2007;61:909-15.

12. Álvarez Sala LA, Suárez C, Mantilla T, Franch J, Ruilope LM, Banegas JR y cols. Estudio Prevencat: control del riesgo cardiovascular en atención primaria. Med Clin (Barc). 2005;124:406-10.

13. Arroyo J, Badia X, De la CH, Díez J, Esmatjes E, Fernández I y cols. Tratamiento de los pacientes con diabetes mellitus tipo 2 en atención primaria en España. Med Clin (Barc). 2005;125:166-72.

14. Molina Aragonés JM. Riesgo cardiovascular, ocupación y riesgos laborales en una población laboral de Catalunya. Med. segur. trab. v.54 n.212. 2008. 